\title{
Open Access Publishing in Mathematics
}

Volker Mehrmann (TU Berlin, Germany), President of the EMS, André Gaul and Laura Simonite (both EMS Publishing House, Berlin, Germany)

The last few years have borne witness to a number of important changes within the scholarly communication sphere that have the potential to radically disrupt research at large, and mathematics in particular.

Researcher and institutional dissatisfaction with traditional journal subscriptions and so-called "Big Deals" have taken the Open Access movement from grassroots activism to politically mandated regulation. The announcement in 2018 of Plan S, an initiative launched by an international consortium of research funders (including the European Commission and the European Research Council) with the explicit aim of making all publicly funded research Open Access, has rapidly accelerated publisher plans to embrace Open Access models of publication.

The European Mathematical Society, in association with a number of other learned societies in the field of mathematics, supports this transition to Open Research. Indeed, mathematics as a discipline has a long-standing culture of liberal Green Open Access policies. We are committed to developing sustainable models for Open Access publishing, as we believe these will come to dominate publishing models in the coming years.

However, this is a transition that should not be taken without due caution and consideration. In many countries in the Northern hemisphere there is a tendency to focus on Gold Open Access as the publishing model of choice a model that requires authors to pay an article processing charge (APC) for publication. This may result in a number of unintended consequences, for example:

- Researchers without funding may be denied access to publish in their journal of choice. This may include researchers from developing economies, or those publishing in underfunded or niche areas of research.

- Journal profitability may become tied to published output, resulting in an inflation in the number of published articles, accompanied by a reduction in the quality of said research.

- APCs are often presented without a breakdown of where costs are incurred in the publication process, which can leave authors and their institutions unsure of the value a publishing house adds to the finished article. 
Society-based publishing houses are not immune to these concerns, nor is the discipline of mathematics as a whole. For this reason it is important that, as the publishing houses of learned societies, we outline a set of guiding principles for our Open Access publishing models to ensure that quality and fairness remain at the heart of our publication programmes. At the same time, publishing houses play an important role in the curation and dissemination of research, so any publishing model must allow for the long-term sustainability of the organisation. We therefore propose the following criteria:

- The quality of publications is paramount and beyond compromise.

- Publications shall be accessible and available in perpetuity.

- Pricing models shall be transparent and fair.

- The publishing house serves the mathematics community, and commits surplus funds to community initiatives.

- The publishing house commits to collaborative relationships with other stakeholders within the mathematics community.

It is with these principles in mind that the publishing house of the European Mathematical Society has begun to investigate sustainable Open Access models for its journal and book portfolio. Over the coming months we will be presenting our findings and inviting feedback from our community of editors, as well as from librarians and researchers in the field.

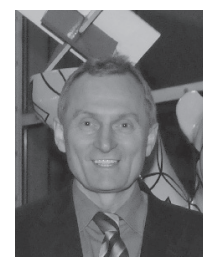

Volker Mehrmann

President

European Mathematical Society

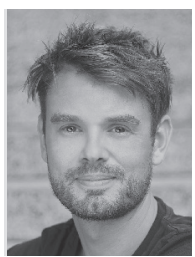

André Gaul

Managing Director

European Mathematical Society Publishing

House

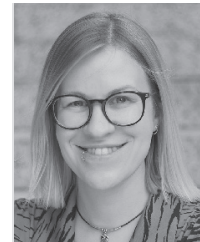

Laura Simonite

Head of Business Development

European Mathematical Society Publishing

House

Press information: for enquiries contact

Laura Simonite at simonite@ems.press. 